\title{
Poor Man's Scaling Approach to the Kondo Effect in a Single Quantum Dot Asymmetrically Attached to Ferromagnetic Leads with Non-Collinear Magnetizations
}

\author{
M. WAWRZYNIAK* \\ Faculty of Physics, Adam Mickiewicz University \\ Umultowska 85, 61-614 Poznań, Poland
}

\begin{abstract}
The poor man's scaling technique is applied to the problem of electronic transport through a single quantum dot (with finite Coulomb repulsion) asymmetrically coupled to electrodes. The considered quantum dot is coupled to ferromagnetic electrodes, whose magnetic moments are non-collinear. The analysis and numerical illustration of spin splitting of the dot level as well as of the Kondo temperature on the coupling asymmetry is presented and discussed.
\end{abstract}

PACS numbers: 75.20.Hr, 72.15.Qm, 72.25.-b, 73.23.Hk

\section{Introduction}

The Kondo effect in quantum dots has been theoretically investigated first for nonmagnetic systems [1], then for magnetic systems with collinear magnetic configurations [2], and finally in magnetic systems with non-collinear magnetic configurations. Recently the problem of the Kondo effect in quantum dots attached to ferromagnetic electrodes with non-collinear magnetic configuration and with asymmetrical coupling to the leads has been addressed [3, 4].

In this work we apply the "poor man's scaling" approach to the Kondo problem in quantum dots coupled asymmetrically to ferromagnetic leads with non-collinear magnetic moments, assuming finite Coulomb repulsion parameter $U$ on the dots.

\section{Model}

We consider a single-level quantum dot attached to two ferromagnetic electrodes, whose magnetic moments are in general non-collinear [3]. The considered

*e-mail: laura@hoth.amu.edu.pl 
system is described by the Hamiltonian

$$
H=\sum_{\alpha} H_{\alpha}+H_{\mathrm{D}}+H_{\mathrm{T}},
$$

where the term $H_{\alpha}=\sum_{\boldsymbol{k} s} \epsilon_{\alpha \boldsymbol{k} s} a_{\alpha \boldsymbol{k} s}^{\dagger} a_{\alpha \boldsymbol{k} s}(\alpha=\mathrm{L}, \mathrm{R})$ describes non-interacting electrons in the left and right electrodes, respectively.

The term $H_{\mathrm{D}}=\sum_{\sigma} \epsilon_{\sigma}^{\mathrm{d}} d_{\sigma}^{\dagger} d_{\sigma}+U n_{\uparrow} n_{\downarrow}$ describes the dot, where $\epsilon_{\sigma}^{\mathrm{d}}$ denotes single-particle energy of the dot's level. Additionally, the Zeeman energy due to external magnetic field $B_{\text {ext }}^{z}$ applied along the dot's quantization axis is taken into account. Here, $d_{\sigma}^{\dagger}\left(d_{\sigma}\right)$ is the creation (annihilation) operator of an electron with spin $\sigma$ on the dot, while $a_{\alpha \boldsymbol{k} s}^{\dagger}\left(a_{\alpha \boldsymbol{k} s}\right)$ describe creation (annihilation) of an electron with spin $s$ and wave-vector $\boldsymbol{k}$ in $\alpha$-th electrode and $n_{\sigma}=d_{\sigma}^{\dagger} d_{\sigma}$. The hopping events between the dot and electrodes are described by the tunneling term

$$
H_{\mathrm{T}}=\sum_{\boldsymbol{k} \alpha} \sum_{s \sigma} T_{\alpha \boldsymbol{k} s} a_{\alpha \boldsymbol{k} s}^{\dagger} R_{\alpha s \sigma} d_{\sigma}+\text { h.c. }
$$

where $T_{\alpha \boldsymbol{k} s}$ denote the tunneling matrix elements, whereas $R_{\alpha s \sigma}$ are elements of the relevant spin rotation matrix. The coupling strength is described by $\Gamma_{\alpha s}(\epsilon)=2 \pi \sum_{\boldsymbol{k}}\left|T_{\alpha \boldsymbol{k} s}\right|^{2} \delta\left(\epsilon-\epsilon_{\alpha \boldsymbol{k} s}\right)$ and is assumed to be energy independent within the energy band extending from $-D$ to $D, \Gamma_{\alpha s}(\epsilon)=\Gamma_{\alpha s}=\Gamma_{\alpha}^{0}\left(1 \pm p_{\alpha}\right)$, where $p_{\alpha}$ denotes spin polarization in the $\alpha$-th electrode. In this paper the analysis is restricted to the case with $p_{\mathrm{L}}=p_{\mathrm{R}}=p$ and the asymmetry in coupling to the leads is taken into account via the asymmetry parameter $0<\gamma<1$, $T_{\mathrm{L} k \boldsymbol{s}}=\gamma T_{\mathrm{R} \boldsymbol{k} s}$. The angle between magnetic moments of the leads is assumed to be $\theta$.

\section{Method and results}

The "poor man's scaling" approach [5] is applied to the system described by the Hamiltonian given by Eq. (1). In order to perform the scaling procedure the dot is assumed to be initially singly occupied, i.e. $-D \ll \epsilon_{\sigma}^{\mathrm{d}} \ll \mu_{\alpha}$ and $\epsilon_{\sigma}^{\mathrm{d}}+U \gg \mu_{\alpha}$, where $\mu_{\alpha}$ denotes the electrochemical potential of the electrodes $\left(\mu_{\alpha}=0\right)$. The next step is to integrate out the high-energy spin-dependent charge fluctuations in the conduction energy band and hence reducing the energy band width. Since this procedure takes into account spin-dependent charge fluctuations the bare dot level $\epsilon_{\sigma}^{\mathrm{d}}$ is renormalized, and the spin-splitting $\delta \epsilon=\epsilon_{\uparrow}^{\mathrm{d}}-\epsilon_{\downarrow}^{\mathrm{d}}$ occurs,

$$
\delta \epsilon=\frac{\Delta}{\pi} p \sqrt{\cos ^{2} \theta / 2+\left(\frac{\gamma^{2}-1}{\gamma^{2}+1}\right)^{2} \sin ^{2} \theta / 2} \times \ln (D / \tilde{D})-g \mu_{\mathrm{B}} B_{\mathrm{ext}}^{z},
$$

where $\Delta=\sum_{\alpha} \Gamma_{\alpha}^{0}$ and $\tilde{D}$ is the energy band cut-off. When $\tilde{D}$ reaches the dot level, the scaling theory for Anderson-like Hamiltonian breaks down. The result given by Eq. (3) is in agreement with that obtained in Ref. [4]. In the case of symmetric coupling, $\gamma=1$ and in the absence of an external magnetic field, the spin splitting of the dot level reaches its maximal value for parallel configuration, namely for $\theta=0$, whereas for the antiparallel configuration, $\theta=\pi$, the spin splitting vanishes. 
Similar result has been obtained in Ref. [6]. If coupling asymmetry is taken into account, $\delta \epsilon$ has maximal values for parallel configuration, while for the antiparallel one it is minimal but does not vanish $(\delta \epsilon \neq 0)$.

Figure 1 shows variation of the dot spin splitting $\delta \tilde{\epsilon}_{\mathrm{d}}=\pi \delta \epsilon / \Delta$ in the absence of external magnetic field with the asymmetry parameter $a^{2}, a=\left(\gamma^{2}-1\right) /\left(\gamma^{2}+\right.$ 1 ), for a few indicated magnetic configurations. It is clearly visible that for the parallel configuration (solid line) splitting is maximal and does not depend on $a^{2}$. For antiparallel configuration (dash-dotted line) the splitting is minimal for all ranges of $a^{2}$ and increases with increasing $a^{2}$ (decreasing $\gamma$ ). For all non-collinear magnetic configurations the spin splitting also increases with increasing coupling asymmetry.

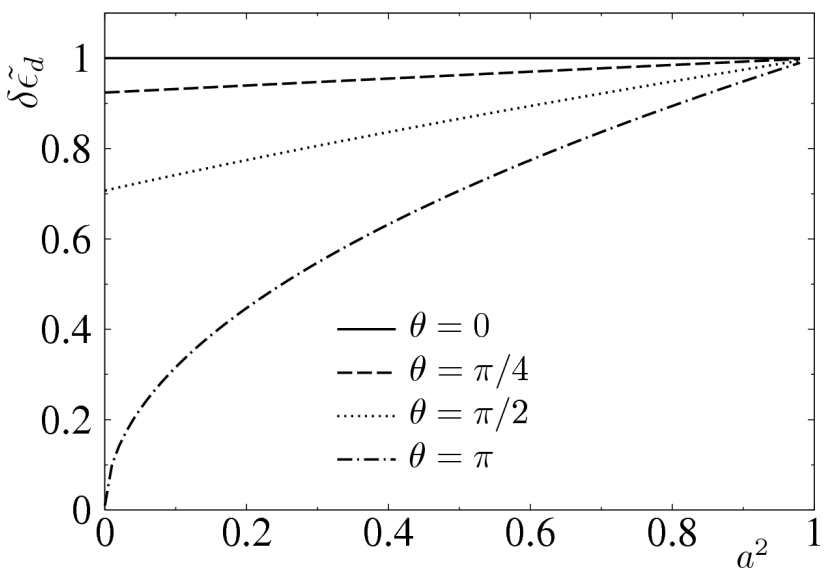

Fig. 1. Variation of the dot spin splitting $\delta \tilde{\epsilon}_{\mathrm{d}}$ in the absence of external magnetic field, calculated as a function of the asymmetry parameter $a^{2}$ for few magnetic configurations indicated in the figure.

Since the scaling technique is no longer valid for Anderson-like Hamiltonian, Eq. (1), the next step in the procedure is to apply the Schrieffer-Wolff transformation [7] in order to obtain the effective $s-d$ Hamiltonian. The scaling procedure is then continued, for the Kondo Hamiltonian. The scaling procedure leads then to the scaling equations for the effective exchange parameters. Finally, solving the scaling equations gives the Kondo temperature [4]:

$$
T_{\mathrm{K}}=\tilde{D} \exp \left(-\frac{\operatorname{arctanh}\left(p \sqrt{\cos ^{2} \theta / 2+a^{2} \sin ^{2} \theta / 2}\right)}{2 j_{0}\left(\rho_{\uparrow}+\rho_{\downarrow}\right) p \sqrt{\cos ^{2} \theta / 2+a^{2} \sin ^{2} \theta / 2}}\right),
$$

where $j_{0}=(\Delta / \pi) U /\left[\left|\epsilon^{d}\right|\left(U+\epsilon^{d}\right)\right]$.

In Fig. 2 the Kondo temperature is plotted vs. coupling asymmetry parameter $a^{2}$ for few magnetic configurations. For the parallel configuration, $\theta=0$, 


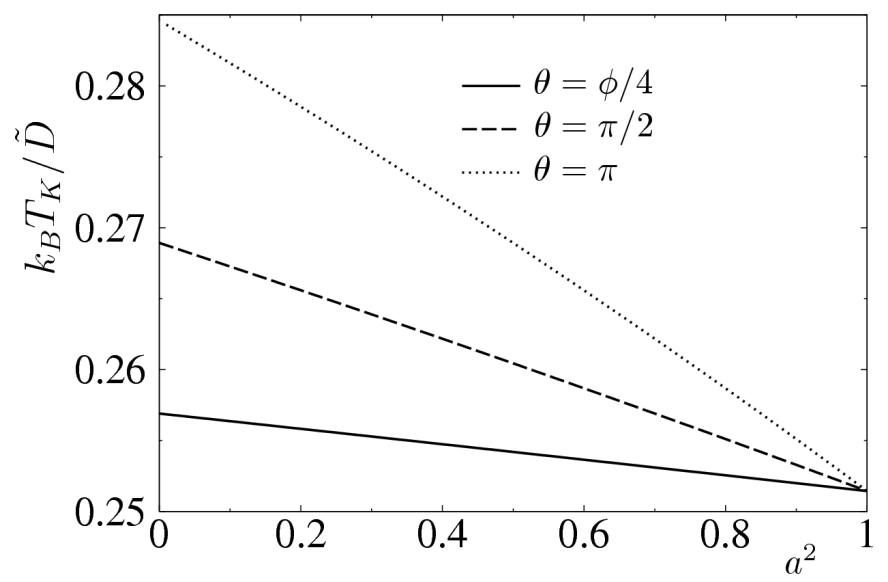

Fig. 2. Kondo temperature $T_{\mathrm{K}}$ as a function of the asymmetry parameter $a^{2}$ for few magnetic configurations indicated in the figure, the other parameters are $p=0.5, U=$ $5 \mathrm{eV}, \epsilon^{\mathrm{d}}=-0.2 \mathrm{eV}, \Delta=0.05 \mathrm{eV}$.

the asymmetry vanishes, see Eq. (4). We observe that in general the coupling asymmetry reduces the Kondo temperature and moreover the Kondo temperature increases with increasing $\theta$ angle, and reaches maximal values for the antiparallel magnetic configuration.

\section{Acknowledgments}

This work was supported by Polish Ministry of Science and Higher Education as a research project in years 2006-2008.

\section{References}

[1] Y. Meir, P.S. Wingreen, P.A. Lee, Phys. Rev. Lett. 70, 2601 (1993).

[2] J. Martinek, Y. Utsumi, H. Imamura, J. Barnaś, S. Maekawa, J. Koenig, G. Schoen, Phys. Rev. Lett. 91, 127203 (2003).

[3] R. Świrkowicz, M. Wilczyński, M. Wawrzyniak, J. Barnaś, Phys. Rev. B 73, 193312 (2006).

[4] D. Matsubayashi, M. Eto, Phys. Rev. B 75, 165319 (2007).

[5] A.C. Hewson, The Kondo Problem to Heavy Fermions, Cambridge University Press, Cambridge UK 1993.

[6] P. Simon, P.S. Cornaglia, D. Feinberg, C.A. Balseiro, Phys. Rev. B 75, 045310 (2007).

[7] J.R. Schrieffer, P.A. Wolff, Phys. Rev. 149, 491 (1966). 